Available online at GSC Online Press Directory

GSC Biological and Pharmaceutical Sciences

e-ISSN: 2581-3250, CODEN (USA): GBPSC2

(RESEARCH ARTICLE)

\title{
Protective effects of vitamin E and grape seed oil against acute hepatorenal ivermectin toxicity in mice: biochemical and histopathological studies
}

El Maghraby Mai Mohsen 1, El Maghraby Nada Mosaad ${ }^{1}$, Salama Ameera Ahmed ${ }^{2}$, Abdlmonem Azza Shawky ${ }^{2}$, Authman Eman Abdellatefe 2, Abdelmohsen Esraa Abdelraouf 2, Eltras Mohamed Abd-Elreheem 2, Barseem Omnia Nasr 2, Awad Shimaa Refat 2, Matter Ahmed Ashraf 2, Abo Sleam Ahmed Magdy 2, Elgozier Esraa Ahmed 2, Elnagar Mohamed Ali ${ }^{2}$, El Bialy Badr El Said ${ }^{3}$ and El Borai Nermeen Borai ${ }^{3, *}$

${ }^{1}$ Undergraduate Student, Fifth Academic Year, Faculty of Veterinary Medicine, University of Sadat City, Sadat City, Egypt. 2 Undergraduate Student, Fourth Academic Year, Faculty of Veterinary Medicine, University of Sadat City, Sadat City, Egypt.

${ }^{3}$ Department of Forensic Medicine and Toxicology, Faculty of Veterinary Medicine, University of Sadat City, Sadat City, Egypt.

Publication history: Received on 23 April 2019; revised on 14 May 2019; accepted on 16 May 2019

Article DOI: https://doi.org/10.30574/gscbps.2019.7.2.0079

\begin{abstract}
Ivermectin (IVM), a broad spectrum anthelmintic drug, is considered a safe drug; however, there are few reports about its toxic effects in particularly at accidental overdose exposure. Therefore, the current study was designed to investigate the potential protective roles of vitamin E (Vit E) and grape seed oil (GSO) against the acute hepatorenal toxicity of IVM in mice. Mice were divided into four equal groups. Control vehicle group was administrated corn oil ( $0.2 \mathrm{ml} / \mathrm{animal})$, IVM group was administrated IVM (6.5 mg/kg b.w.), Vit E+IVM group was administrated Vit E (200 mg/kg b.w.) plus IVM and GSO+IVM group was administrated GSO (0.2 ml/animal) plus IVM. All treatments were orally administrated daily for 3 weeks while IVM is administered as a single oral dose one day before the end of the experiment. The results revealed that IVM induced significant elevation in serum ALT and AST activities; urea and creatinine levels without any significant change in glucose level. No marked histopathological alterations were observed in hepatic tissue with several pathological alterations in the kidneys of IVM- intoxicated mice. However, pretreatment of mice with either Vit E or GSO ameliorated the IVM- induced biochemical and histopathological alterations. In conclusion, Vit E and GSO may provide a similar promising protective value against IVM acute hepatorenal intoxication.
\end{abstract}

Keywords: Ivermectin; Vitamine E; Grape seed oil; Hepatorenal toxicity; Histopathology

\section{Introduction}

Pesticides including insecticides, herbicides, fungicides, rodenticides, and others, are being used extensively throughout the world for controlling industrial, agricultural, domestic and public health pests [1]. The indiscriminate use of pesticides causes tremendous environmental pollution and health hazards to target and non-target organisms [2]. Among them, ivermectin is frequently used to control parasitic infestations in many animal species [3].

Ivermectin (IVM) is semisynthetic macrocyclic lactone produced from Streptomyces avermitilis. It has antiparasitic effects against gastrointestinal and lung nematodes, arthropods and certain ectoparasites [4]. It is considered the drug of choice for treatment of Onchocerciasis, Ascariasis and Enterobiasis in human [5].

\footnotetext{
${ }^{*}$ Corresponding author

E-mail address: nermeenborai@yahoo.com
} 
The antiparasitic action of IVM is referred to its agonist effect on glutamate and gama-aminobutyric acid (GABA)-gated chloride channels, which cause influx of chloride causing CNS inhibition and paralysis [6]. IVM is metabolized primary via the oxidative pathway in liver by microsomal cytochrome P450, its metabolites are excreted mainly in feces and to less extent in urine $[7,8]$.

Ivermectin poisoning causes CNS depression and sometimes coma resulting in death [6]. Beside its neural effects, IVM has toxic effects on liver and kidney at either therapeutic or toxic doses in albino rats [9]. Cumulative evidences have shown that pesticide poisoning disrupts redox homeostasis along with induction of oxidative damage. The disruption of redox homeostasis during IVM poisoning is due to the increased production of reactive oxygen species (ROS) and the reduction in endogenous antioxidant defense system $[10,11]$.

Vitamin E is a lipid soluble antioxidant naturally presents in all biological systems and protects cell membranes against lipid peroxidation (LPO) which is induced by overproduction of ROS and reactive nitrogen species (RNS). It acts as membrane stabilizer and a chain-breaking antioxidant that prevents propagation of lipid peroxidation reactions in cellular membrane $[12,13]$. Therefore, several in vivo and in vitro studies confirmed the protective effect of Vit $\mathrm{E}$ against different pesticides toxic insults [14-17].

Grape (Vitis vinifera), one of the most widely consumed fruits, have been of interest worldwide because of the nutritional properties of the natural product (raw and dried fruit) and the pharmaceutical properties of its derivatives, such as peel and seed extracts [18]. Grape seed oil (GSO) is a by-product of wine making process and has gained use as culinary oil because of its high levels of phenolic compounds, fatty acids, vitamins, and phytosterols. GSO has a powerful antiatherosclerotic, anti-inflammatory, antioxidant, antitumor and anti-apoptotic $[19,20]$ effects.

In view of the clinical importance of IVM in animals and humans and the dearth of literature on the host toxicity, the current study was designed to investigate the potential protective roles of vitamin $\mathrm{E}$ and grape seed oil against the acute hepatorenal toxicity of IVM in mice.

\section{Material and methods}

\subsection{Experimental animals}

Forty mature healthy female mice (30-35 g) were donated from Department of Husbandry and Animal Wealth Development, Faculty of Veterinary Medicine, University of Sadat City, Egypt. Mice were housed in polypropylene cages with mesh wire tops and kept in a natural ventilated room $\left(23 \pm 3{ }^{\circ} \mathrm{C}, 45-60 \% \mathrm{RH}, 12 \mathrm{~h}\right.$ light/ $12 \mathrm{~h}$ dark cycle $)$, provided with standard commercial diet and clean tap water ad libitum. Mice were acclimatized to the laboratory environmental conditions for 2 weeks prior to the start of experiment. The study was ethically approved by the International Animal Care and Use Committee IACUC, Faculty of Veterinary Medicine, University of Sadat City.

\subsection{Chemicals}

Ivermectin (Avimec, Arab Veterinary Industrial Company) was purchased from local Veterinary Pharmacy. Vitamin E (400 mg oily capsules, Pharco Pharmaceutical Company) was purchased from local pharmacy. Grape seeds oil (Vitis vinifera seed oil) was purchased from Harraz Group for Medicinal Plants, Cairo, Egypt.

Diagnostic kits for assaying serum glucose level, ALT and AST activities were purchased from Spinreact Company, Spinreact Ctra. Sta. Coloma, Girona, Spain. Diagnostic kits for assaying serum urea and creatinine levels were purchased from Biodiagnostics Company, Cairo, Egypt. Other utilized chemicals were of analytical grade and commercially available.

\subsection{Experimental design and animal grouping}

Mice were weighed, randomly allocated into four equal groups. Control vehicle group (corn oil) was orally administrated corn oil ( $0.2 \mathrm{ml} /$ animal) daily for 3 weeks. IVM group was administrated IVM as a single oral dose, 6.5 $\mathrm{mg} / \mathrm{kg}$ b.w., 1/5 oral LD50 on the last day of the experiment. Vit E+IVM group was orally administrated Vit E, $200 \mathrm{mg} / \mathrm{kg}$ b.w. [21] plus IVM administration. GSO+IVM group was orally administrated GSO, $0.2 \mathrm{ml} /$ animal [22] plus IVM administration. The dose of IVM was selected according to oral LD50 in mice, $33 \mathrm{mg} / \mathrm{kg} \mathrm{b} . \mathrm{w}$. [23]. All treatments were orally administrated daily for 3 weeks while IVM is administered as a single oral dose one day before the end of the experiment. 


\subsection{Samples collection}

At the end of the experiment and after 24 hours of IVM administration, mice were fasted overnight, anaesthetized using diethyl ether and sacrificed for samples collection. Blood samples were collected in clean and dry glass centrifuge tubes for serum collection and then stored at $-20^{\circ} \mathrm{C}$ for further serum biochemical analysis. Liver and kidney of each mice were collected and fixed in $10 \%$ neutral buffered formalin solution for the histopathological investigation.

\subsection{Serum biochemical analysis}

Serum ALT and AST activities [24, 25]; glucose [26], urea [27] and creatinine [28] levels were estimated using commercial kits following the manufacturer's instructions.

\subsection{Histopathological examination}

Following necropsy, tissue specimens from liver and kidneys were collected and rapidly fixed in $10 \%$ neutral buffered formalin solution. The fixed specimens were trimmed, washed, dehydrated in ascending grades of ethyl alcohol, cleared in methyl benzoate and processed through the conventional paraffin embedding technique. 3-5 $\mu \mathrm{m}$ sections were obtained from paraffin blocks using microtome (LEICA RM 2135) then routinely stained by hematoxylin and eosin (H \& E) stain according to Bancroft et al., [29]. Stained slides were microscopically analyzed using light microscopy. Histopathological Photos were photographed using a digital Leica photomicroscope (LEICA DMLB Germany).

\subsection{Statistical analysis}

Values are presented as mean \pm standard error (SE). Statistical significance of data was determined by one-way ANOVA (Analysis of Variance) followed by Duncan's Multiple range test for post hoc analysis. All statistical analyses were performed using SPSS (Statistical Package for Social Sciences) Version 16 released on 2007.

\section{Results}

\subsection{Effects on animals' general health conditions}

No mortalities were recorded along the period of the experiment. Mice of corn oil group did not exhibit any clinical manifestations. However, after a single oral dose administration of IVM $(6.5 \mathrm{mg} / \mathrm{kg}$ b.w.) alone or after Vit E or GSO treatment, mice showed short time of excitatory symptoms accompanied by abdominal respiration followed by depressive stage. These signs were more predominant in IVM- intoxicated mice and relieved after short period of insecticide administration.

\subsection{Serum biochemical findings}

\subsubsection{Serum ALT and AST activities}

Oral administration of a single oral dose of IVM $(6.5 \mathrm{mg} / \mathrm{kg}$ b.w.) induced significant elevations in serum ALT and AST activities $(P<0.05)$ compared to corn oil treated group. However, pretreatment with either Vit $\mathrm{E}(200 \mathrm{mg} / \mathrm{kg}$ b.w. $)$ or GSO ( $0.2 \mathrm{~g} / \mathrm{mice})$ daily for three weeks before IVM intoxication significantly reduced serum ALT and AST activities to be within normal corn oil values (Fig. 1).

\subsubsection{Serum glucose level}

Serum glucose level showed insignificant changes between all treated groups at $P<0.05$ (Fig. 1).

\subsubsection{Serum urea and creatinine levels}

Regarding to corn oil group, IVM- intoxicated group showed significant increase $(P<0.05)$ of serum urea and creatinine levels. Pretreatment with either Vit E or GSO three weeks before IVM- administration significantly reduced the elevated urea and creatinine levels and restored all the values to the normal limits (Fig. 1). 


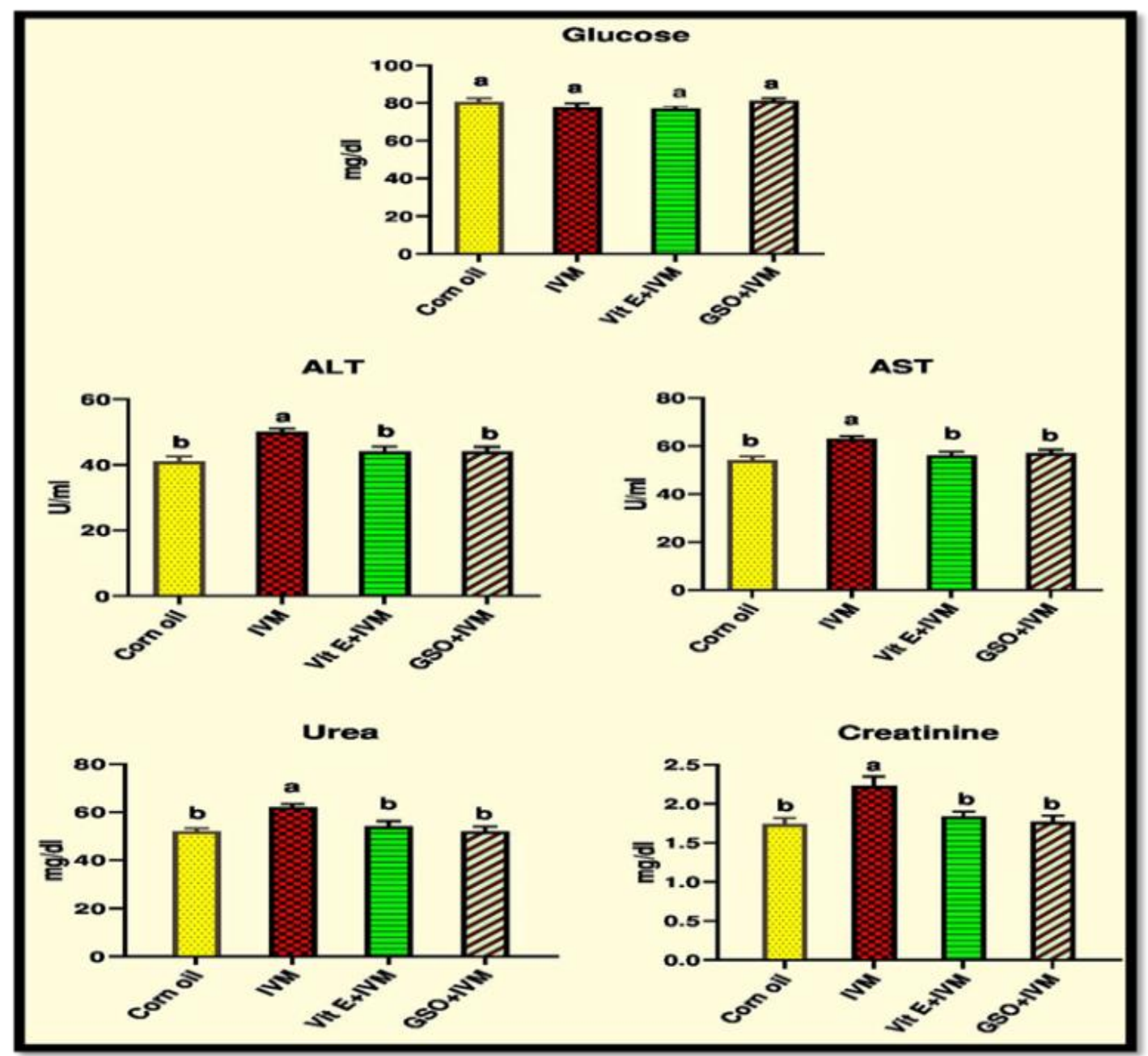

Figure 1 Serum biochemical parameters of the different treated groups. Different letters means significant differences at $(\mathrm{P}<0.05)$. IVM: ivermectin; Vit E: vitamin E; GSO: grape seed oil

\subsection{Histopathological findings}

Hitopathological examination revealed no marked pathological changes in the liver of all treated groups.

The kidneys of corn oil- administrated mice showed no pathological changes (Fig. 2a). However, IVM- intoxicated mice showed various pathological changes in the kidneys, including intertubular edema and hemorrhage, edema in the interstitial tissue and around glomeruli, vacuolation of glomerular tuft, narrowing of Bowman's space, coagulative necrosis and karyorrhexis in the lining epithelium of renal tubules (Fig. 2b). Pretreatment with Vit E improved the renal picture as presented by minimal edema and slight cloudy swelling in the epithelium lining of some renal tubules (Fig. 2c). Similarly, GSO administration enhanced the renal architecture with only mild hydropic degeneration in lining epithelia of some renal tubules (Fig. 2d). 


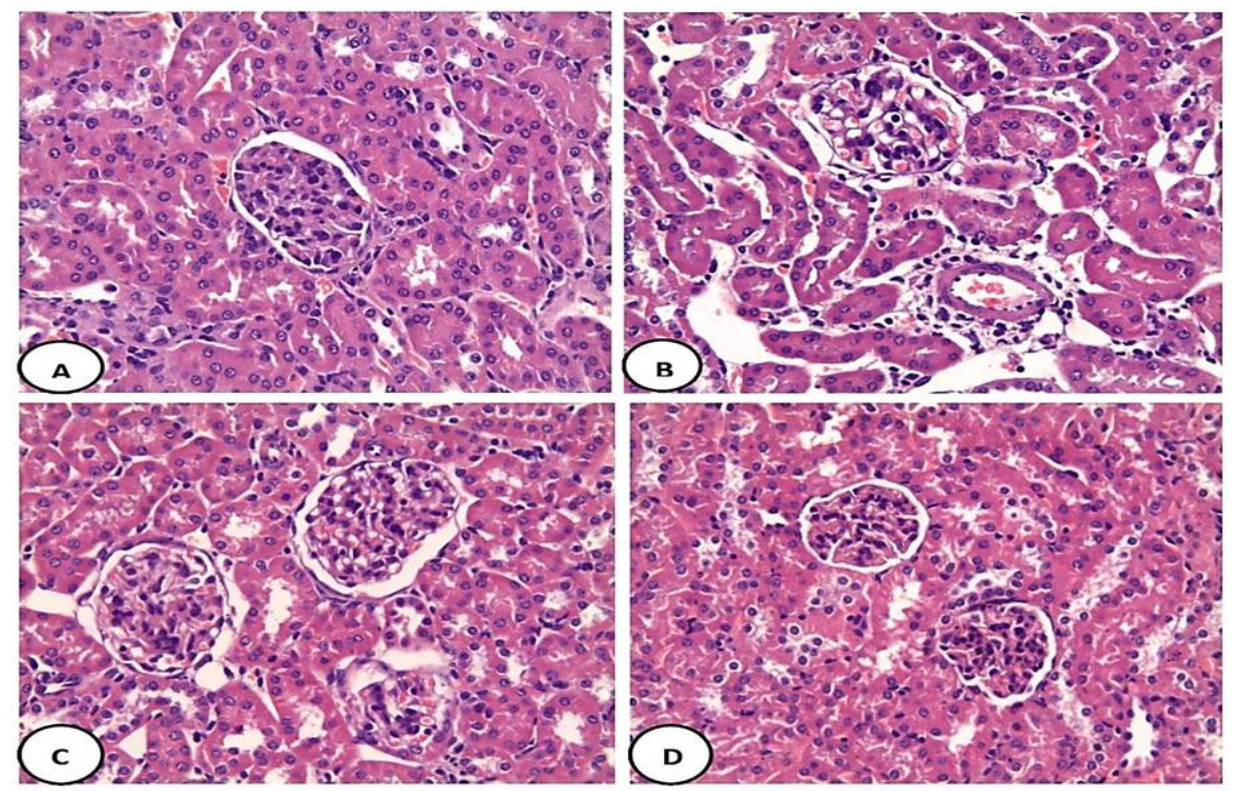

Figure 2 Representative photomicrographs of kidney of mice in different groups (H\&E, X20).

A: Corn oil group: showing no pathological changes. B: IVM group: showing intertubular edema and hemorrhage, edema in the interstitial tissue and around glomeruli, vacuolation of glomerular tuft, narrowing of Bowman,s space, coagulative necrosis and karyorrhexis in the lining epithelium of renal tubules. C: Vit E+IVM group: showing minimal edema and slight cloudy swelling in the lining epithelium of some renal tubule. D: GSO+IVE group: showing mild hydropic degeneration in the lining epithelia of some renal tubules.

\section{Discussion}

Ivermectin is one of the most frequently used drugs in pharmacotherapy of parasitic diseases in domestic and wild animals. It is widely recommended for field applications due to easy route of administrated and potent larvicidal activity [30]. However, ivermectin and other avermectins very often produce side-effects in hosts. Cumulative evidences have confirmed the implication of oxidative stress in IVM- induced toxicity [10,31]. Therefore, considerable attention has been gained toward using of natural antioxidants to overwhelm the oxidative damages of pesticides. In the light of this, the current study aimed to evaluate the potential protective roles of vitamin $\mathrm{E}$ and grape seed oil against the hepatorenal toxicity of IVM in mice.

The liver and kidneys have vital role in the metabolism and excretion of xenobiotics, consequently leading to toxic cellular damages [32]. In the current study, estimation of liver and kidney biomarkers with histopathological examination has curial value for assessment of the hepatorenal toxicity of IVM in mice and help in evaluation of the potential ameliorative role of the Vit E and GSO.

Ivermectin caused significant elevations in serum ALT and AST activities in mice, this result is in agreement with Qureshi [10], who recorded increase in serum ALT and AST activities after IVE single IP injection (10 and $15 \mathrm{mg} / \mathrm{kg}$ b.w.) in rats. The increase in hepatic function enzyme activities is considered to be indicators for cytotoxicity and disruption of cellular membrane integrities leading to leakage of these enzymes and their release into blood [33]. Also, the biotransformation of IVE to more electrophilic and oxidative metabolites induces oxidant/antioxidant disturbance leading to several biochemical and histopathological changes [10, 34].

Unexpectedly, there were no marked histopathological changes in hepatic tissues in all treated groups. Chiu and Lu [35] demonstrated that a single oral dose of IVE results in peak plasma levels at 4 to $8 \mathrm{hrs}$ post oral administration, with a half-life of $24 \mathrm{hrs}$. This means that oral single dose of IVE rapidly absorbed and eliminated. This may illustrate the recorded renal pathological changes without marked hepatic changes that probably occurred later in time.

Regarding to serum renal biomarkers, significant increase in serum urea and creatinine levels were recorded in IVMintoxicated mice. The serum biochemical changes were correlated with histopathological alteration in kidney tissue. In consistent with our finding, Arise and Malomo [36] recorded significant urea and creatinine levels in rats orally administered a human therapeutic dose of IVM; daily for 15 days. Similarly, rats subcutaneously injected either therapeutic or double therapeutic doses of IVM, once weekly for eight weeks increased serum urea, creatinine and uric acid levels in association with changes in renal histologic structure [37] 
Serum urea and creatinine are renal biomarkers used to evaluate kidney functions [38] and hence, elevation of their levels in IVM- intoxicated rats reflects its nephrotoxic effects which may be attributed to the reduction of glomerular filtration rate and the dysfunction of the kidney tubules [39]. Also, IVM is known to cause inhibition of renal excretion of substances that are substrates of $p$-glycoprotein [40] and induction of oxidative renal damage [11] leading to retention on urea and creatinine in blood.

In this study Vit E ameliorated serum biochemical and histopathological changes induced by IVM as indicated by significant reduction in serum ALT, AST activities and urea, creatinine levels associated with improvement of renal histologic structure. Similarly, previous studies proved the hepatorenal protective effect of Vit E against abamectin [15] and fipronil [17] toxicities. Vitamin E is well known to be potent natural antioxidant; it has important functional properties in membranes lipoprotein, it exerted an efficient antioxidant role by neutralization of ROS and up-regulation of antioxidant enzymes and reduction in lipid peroxidation $[41,42]$.

Pretreatment of mice with GSO three weeks before IVE intoxication returned serum liver and kidney biochemical changes to be within normal levels with improvement of renal histological pictures. The same findings were recorded either after grape seed oil and extract administrations against hepatorenal toxicity of diazinone [22], cisplatin [43] and fumonisin B1 [44]. The protective effects of GSO are referred to its polyphenolic contents that have free radicals scavenging properties [43, 44].

\section{Conclusion}

In the light of the above findings, we can conclude that administration of IVM as a single oral dose induced changes in serum liver and kidney biomarkers accompanied by histopathological alterations in the kidney of mice. Pretreatment of mice either with Vit E or GSO three weeks before IVM intoxication markedly and equally reversed these alterations referring to their antioxidant defensive mechanisms.

\section{Compliance with ethical standards}

\section{Acknowledgments}

The authors gratefully acknowledge and thank Dr. Anis Zaid (Assistant Professor of Pathology Faculty of Veterinary Medicine, University of Sadat City) for his valuable help in the histopathological examination.

\section{Disclosure of conflict of interest}

The authors declare that there are no conflicts of interest.

\section{Statement of ethical approval}

This study was ethically approved by the International Animal Care and Use Committee IACUC, Faculty of Veterinary Medicine, University of Sadat City.

\section{References}

[1] Bjorling-Poulsen M, Andersen HR and Grandjean P. (2008). Potential developmental neurotoxicity of pesticides used in Europe. Environmental Health, 7(50), 1-23.

[2] Noaishi MA, Aify MM and Allah AA, (2013). Study the inhalation exposure effect of pesticide mixture in the white rat. Nature and Science, 11(7), 45-54.

[3] Chabala JC, Mrozik H, Tolman RL, Eskola P, Lusi A, PetersonLH, Woods MF and Fisher MH. (1980). Ivermectin, a new broad- spectrum antiparasitic agent. Journal of Medical Chemistry, 23, 1134-1136.

[4] Roberson FL. (1988). Antinematodal drugs. In: Veterinary Pharmacology and Therapeutics. Booth, N.H. and McDonald, L.E. (eds), 3rd Ed. Iowa State University Press, Ames, Iowa. pp. 340-345. 48. Roder, J.D. 1998. An overview of ivermectin toxicosis. Veterinary and Human Toxicology, 40, 369-370.

[5] Gonzalez P, Gonzalez FA and Ueno K. (2012). Ivermectin in human medicine, an overview of the current status of its clinical applications. Current Pharmaceutical Biotechnology, 13, 1103-1109.

[6] Ottesen EA and Campbell WC. (1994). Ivermectin in human medicine. Journal of Antimicrobial Chemotherapy, 34(2), 195-203. 
[7] Zeng Z., Andrew NW, Arison BH, Luffer-Atlas D and Wang RW. (1998). Identification of cytochrome P4503A4 as the major enzyme responsible for the metabolism of ivermectin by human liver microsomes. Xenobiotica, 28, 313-321.

[8] Plumb DC. (2008). Plumbs veterinary drug handbook. Blackwell publishing. 6th Edi. Stockholm. P, 508.

[9] Ashang BU. (2009). Effect of therapeutic and toxic doses of ivermectin (Mectizan) on total serum proteins and hepatic enzymes of wistar albino rats. International Journal of Biological and Chemical Sciences, 3, $142-147$.

[10] Qureshi S. (2013). Biochemical toxicity of ivermectin in Wistar albino rats. American-Eurasian Journal of Toxicological Sciences, 5 (1), 15-19.

[11] El-Ashmawy IM. (2019). Effects of ivermectin on the brain and kidney and its interaction with p-glycoprotein inhibitor (Verapamil) in rats. Research Journal of Pharmaceutical, Biological and Chemical Sciences, 10(2), 402410.

[12] Kamal-Eldin A and Appelqvist LA. (1996).The chemistry and antioxidant properties of tocopherols and tocotrienols. Lipids, 31(7), 671-701.

[13] Clarke MW, Burnett JR and Croft KD. (2008). Vitamin E in human health and disease. Critical Reviews in Clinical Laboratory Science, 45(5), 417-50.

[14] Saxena R, Garg P and Jain DK. (2011). In vitro anti-oxidant effect of vitamin E on oxidative stress induced due to pesticides in rat erythrocytes. Toxicology international, 18(1), 73.

[15] Magdy BW, Amin AS and Rana SS. (2016). The Egyptian German Society for Zoology Ameliorative effect of antioxidants (vitamins $\mathrm{C}$ and $\mathrm{E}$ ) against abamectin toxicity in liver, kidney and testis of male albino rats. Journal of Basic and Applied Zoology, 77, 69-82.

[16] Selim A, khalaf MM, Gad AM and Abd El-Raouf OM. (2017). Evaluation of the possible nephroprotective effects of vitamin $\mathrm{E}$ and rosuvastatin in amikacin-induced renal injury in rats. Journal of Biochemical and Molecular Toxicology, e21957.

[17] Abdel-Daim MM. and Abdeen A. (2018). Protective effects of rosuvastatin and vitamin E against fipronil-mediated oxidative damage and apoptosis in rat liver and kidney. Food and Chemical Toxicology, 114, 69-77.

[18] Bail S, Stuebiger G, Krist S, Unterweger H and Buchbauer G. (2008). Characterisation of various grape seed oils by volatile compounds, triacylglycerol composition, total phenols and antioxidant capacity. Food Chemistry, 108(3), 1122-1132.

[19] Yassa N, Beni HR and Hadjiakhoondi A. (2008). Free Radical Scavenging and Lipid Peroxidation Activity of the Shahani Black Grape. Pakistan Journal of Biological Sciences, 11(21), 657-661.

[20] Garavaglia J, Markoski MM, Oliveira A and Marcadenti A. (2016). Grape Seed Oil Compounds: Biological and Chemical Actions for Health. Nutrition and Metabolic Insights, 9, 59-64.

[21] Maheswari E, Saraswathy GR, and Santhranii T. (2015). Influence of Vitamin E on Hepatotoxicity and Oxidative Stress. International Journal of Research in Pharmacy and Biosciences, 2 (3), 30-38.

[22] Al-Attar MS. (2017). Antimutagenic effect of grape seed extracted oil on diazinon induced genotoxicity in albino mice. Iraqi of Cancer and Medical Genetics, 10 (1), 56-62.

[23] Novik TS, Baru RV, Riabova VA, Chukina SI, Krugliak EB, Mosin VA and Driniaev VA. (2001). Acute toxicity of aversectin C: various routes of administration and dosage forms. Eksperimental'naia i klinicheskaia farmakologiia, 64(2), 64-6.

[24] Murray R. (1984a). Alanine aminotransferase. In: Clinical Chemistry, Eds., Kaplan A, AL Peace, the C. V. MosbyCo., St Louis, Toronto, Princeton, 1088-1090.

[25] Murray R. (1984b). Aspartate aminotransferase. In: Clinical Chemistry, Eds., Kaplan, A. and AL Peace, the C.V. Mosby Co., St Louis, Toronto, Princeton.,1112-1116.

[26] Young DS. (2001). Effects of disease on Clinical Lab. Tests, 4th ed Washington, DC: AACC press.

[27] Fawcett JK and Soctt JE. (1960). A rapid and precise method for the determination of urea. Journal of Clinical Pathology, 13(2), 156 - 159.

[28] Schirmeister J. (1964). Determination of creatinine level. Deutsche Medizinische Wochenschrift. 89, 1940-1947. 
[29] Bancroft D, Stevens A and Turner R. (1996). Theory and practice of histological techniques. 4th edition, Churchill Livingstone, Edinburgh, London, Melbourne.

[30] Sharma SP and Siddiqui AA. (1996). Efficcacy of three anthelmintics in experimentally induced stariosis in lambs". Journal Veterinary Parasitology, 10, 159-163.

[31] Hosseini-Omshi FS, Abbasalipourkabir R, Abbasalipourkabir M, Nabyan S, Bashiri A and Ghafourikhosroshahi A. (2018). Effect of vitamin A and vitamin C on attenuation of ivermectin-induced toxicity in male Wistar rats. Environmental Science and Pollution Research, 25(29), 29408-29417.

[32] O’Shaunessy K. (2010). Principles of Clinical Pharmacology and Drug Therapy. In: Warrel D., Cox T., Firth J., editors. Oxford Textbook of Medicine. 5th ed. Vol. 1. Oxford University Press; Oxford, UK.1450-1476.

[33] Jimoh FO and Odutuga AA. (2001). Changes in the activities of some diagnostic enzymes in some rat tissuses following the consumption of thermally oxidized ground nut oil. Nigerian Journal of Biochemistry and Molecular Biology, 16, 173-176.

[34] Sutherland IH and Campbell WC. (1990). Development, pharmacokinetics and mode of action of ivermectin. ActaLeiden, 59, 161-168.

[35] Chiu SHL and Lu AYH. (1989). Metabolism and tissue residues, p. 131-143. In W. C. Campbell (ed.), Ivermectin and abamectin. Springer-Verlag, New York.

[36] Arise RO. and Malomo SO. (2009). Effects of ivermectin and albendazole on some liver and kidney function indices in rats. African Journal of Biochemistry Research, 3 (5), 190-197.

[37] Elzoghby RR, Amin A, Hamouda FA and Ali A. (2015). Toxicological and pathological studies of Ivermectin on male albino rats. Journal of American Science, 11, 73-83.

[38] Inui KI, Masuda S and Saito H. (2000). Cellular and molecular aspects of drug transport in the kidney. Kidney International, 58(3), 944-58.

[39] Walmsley RN and White GH. (1994). A guide to diagnostic clinical chemistry. 3rd Ed., Blackwell. Scientific Publication, London.

[40] Fricker A, Cutmann H, Droulle A, Drewe J and Miller DS. (1999). Epithelial transport of anthelmintic ivermectin: a novel model of isolated proximal kidney tubules. Pharmaceutical Research, 16, 1570-1575.

[41] Feng Y, Huang X, Duan Y, Fan W, Duan J, Wang K, Geng Y, Ouyang P, Deng Y, Chen D and Yang S. (2018). The Effect of Vitamin E and Metallothionein on the Antioxidant Capacities of Cadmium-Damaged Liver in Grass Carp Ctenopharyngodon idellus. BioMed Research International, 2018, 7935396.

[42] Akyüz F, Kara Y, Kar F, Hacıoğlu C and Burukoğlu Dönmez D. (2018). Protective Effect of Vitamin E and N-Nitro L-Arginine Methyl Ester (L-NAME) on Cigarette Induced Kidney Damage. Turkish Journal of Life Sciences, 3(1), 205-209.

[43] Yousef MI, Saad AA and El-Shennawy LK. (2009). Protective effect of grape seed proanthocyanidin extract against oxidative stress induced by cisplatin in rats. Food and Chemical Toxicology, 47(6), 1176-1183.

[44] El-Adawi H, El-Azhary D, Abd El-Wahab A, El-Shafeey M and Abdel-Mohsen M. (2011). Protective effect of milk thistle and grape seed extracts on fumonisin B1 induced hepato- and nephro-toxicity in rats. Journal of Medicinal Plants Research, 5(27), 6316-6327.

\section{How to cite this article}

El Maghraby MM, El Maghraby NM, Salama AA, Abdlmonem AS, Authman EA, Abdelmohsen EA, Eltras MA, Barseem ON, Awad SR, Matter AA, Abo Sleam AM, Elgozier Esraa A, Elnagar Mohamed A, El Bialy BE and El Borai NB. (2019). Protective effects of vitamin $\mathrm{E}$ and grape seed oil against acute hepatorenal ivermectin toxicity in mice: biochemical and histopathological studies. GSC Biological and Pharmaceutical Sciences, 7(2), 87-94. 